\title{
Quantifying the effect of home visit occupational therapy on the quality of life of elderly individuals
}

\author{
Miyuki Imanishi ${ }^{1}$, Hisao Tomohisa ${ }^{2}$, Kazuo Higaki $^{3}$ \\ ${ }^{1}$ Research Institute of Rehabilitation Sciences, Osaka Prefectural University, Osaka, Japan \\ ${ }^{2}$ Department of Psychiatry, Kyoto University Hospital, Kyoto, Japan \\ ${ }^{3}$ Department of Community Health, Osaka Prefectural University, Osaka, Japan
}

\begin{abstract}
:
Purpose: Japan is now the world's first 'super-ageing' society. We analysed the effect of occupational therapy (OT) sessions in addressing issues related to the elderly population, in comparison with other services.

Methods: We studied 136 elderly patients receiving at-home care. Seventy-four patients received weekly OT and 62 did not. We assessed quality of life (QOL) and its trajectory over 1 year using the Philadelphia Geriatric Center Morale Scale (PGC) to quantify changes in QOL and the Function Independence Measure (FIM) to measure changes in activities of daily living.

Results: Patient progress fell within five different trajectories, which was influenced by psychosocial factors. OT correlated with significantly greater improvement in PGC and FIM scores than other home care services.

Conclusion: There is a significant benefit associated with OT and is expected to become increasingly more important in the growing elderly population. Tailoring of OT will be required to benefit those trajectory groups that showed unsatisfactory outcomes.
\end{abstract}

Keywords: home-visit occupational therapy, quality of life, elderly individuals

(Asian J Occup Ther 13: 1-6, 2017)

\section{Introduction}

Japan is in the middle of a unique and unprecedented demographic change; the percentage of citizens 65 years and older has now risen to $23 \%$ of the total population [1] and will increase to $33 \%$ by 2030 [2]. This has been attributed to the tendency toward a lower total fertility rate as rural populations dwindle when younger generations move to cities, where large families are impractical due to limited space, high cost of living and inadequate childcare opportunities for working mothers. Another component is the increasing longevity of the elderly population and the growing tendency of their children to live away from them [3].

Received: 3 July 2015, Accepted: 1 September 2016

Corresponding to: Miyuki Imanishi, Research Institute of Rehabilitation Sciences, Osaka Prefectural University, Habikino 3-7-30, Habikino-shi, Osaka, Japan e-mail: miyuki726@kcn.jp

(C2017 Japanese Association of Occupational Therapists
The relative isolation and potential vulnerabilities of the elderly age group were brought to the nation's attention by the Great East Japan earthquake, followed by a tsunami, on 11 March 2011. Elderly patients were over-represented in the number of citizens requiring emergency care and shelter [4].

To address problems facing ageing societies, the Japanese government has adopted measures to reduce medical expenses among the elderly, including the establishment of a long-term care insurance system and the revision of medical fees. This has been accompanied by an emphasis on in-home care, with a shift 'from care by family' to 'care by society' $[5,2,6]$

This shift has, in turn, focused attention on the issue of quality of life (QOL) among those receiving in-home care. Kawai [7] points out that the social norms of a super-ageing society tend to regard the elderly as a social problem that needs to be addressed, but it is questionable whether the elderly can live happily knowing that they are considered a social problem. Washida [8] asserts that humans cannot live without recognizing 
meaning in their lives. This in turn requires that we should strive to organize and administer occupational therapies that prevent elderly people from losing meaning in their lives and help them maintain or improve QOL.

Studies to date on the QOL of the elderly have been conducted from various viewpoints. Lawton [9] defined QOL in this population as 'subjective well-being' and created the Philadelphia Geriatric Center Morale Scale (PGC) (see Appendix) in 1972 to measure QOL among the elderly. The PGC is still used worldwide. In another study, Liang and Bollen [10] described factors affecting QOL in the elderly, including sex, race, marital status, health condition, employment situation, socioeconomic status and social activities. Koyano [11] asserted that being in good health and maintaining close relationships with others helps improve QOL. These studies primarily targeted healthy elderly people and did not specifically investigate elderly persons receiving occupational therapy (OT). Nevertheless, their findings serve as a benchmark for future consideration of geriatric QOL. Analyzing the differences in QOL between elderly persons receiving home care with or without OT may help therapists to tailor OT and improve patient QOL.

To assess these differences in home care, we conducted a prospective analysis of subjects who started to use home care services with and without OT over 1 year.

\section{Materials and Methods}

\subsection{Design}

The study was conducted under a prospective cohort design based on age-related physical function and QOL score in 200 patients aged 65 to 84 years who had begun to use home care services.

\subsection{Study Participants and Activities}

The study lasted from May 2013 to May 2014 and included patients from 12 visit care stations in three prefectures (Kyoto, Osaka and Nara Prefectures) in central Japan. Participants in the OT cohort were scheduled to commence receiving home care visits that consisted of weekly $1 \mathrm{~h}$ visits by an occupational therapist or nurse, who engaged the patient in exercise therapy [activities of daily living (ADL) practice], OT (cooking, gardening, crafts, music activities, going out-of-doors, practice in operating mobile, etc.), proposals of leisure activities or volunteer activities, counselling and home exercise guidance.

Participants in the non-OT cohort received 1-5 weekly home visits totalling $60-300 \mathrm{~min}$ by a nurse or healthcare professional who assisted the patient with cleaning, laundry, shopping, bathing, eating and medication management.

Setting

All 12 care stations provide home visit care service. In five locations, professional caregivers help with ADL, bathing, housework, shopping and conversation. The patients served by these five care stations were eligible for the study as part of the non-OT cohort. In the other seven locations, nurses or occupational therapists provide medical services including blood pressure measurement, physical therapy, family therapy, OT and prescription management. The patients served by these seven care stations were eligible for the study as part of the OT cohort.

\subsection{Inclusion/exclusion criteria}

Inclusion criteria included intact cognitive function and communication skills, and a stable general condition that allowed participation. Patients scoring less than 24 points on the Mini-Mental State Examination were excluded.

\subsection{Data collection}

Physical function and QOL scores were investigated in the patients' homes at intervals of $90 \pm 10$ days using the Functional Independence Measure (FIM) and the PGC Morale Scale. The PGC Morale Scale, used to measure QOL, comprises 17 questions (see Appendix). A normal score is $11.97 \pm 3.57$, derived from a population of healthy community-dwelling elderly people in Japan. The main outcome of this study was the PGC Morale Scale score.

Patient ADL was assessed using the FIM. The FIM evaluates movement and cognitive function in 18 items and 7 steps and is mainly used at rehabilitation clinic sites, both in Japan and abroad. The FIM score was the secondary outcome of this study. Both tests were administered by specialists, such as occupational therapists. The questionnaire for the PGC Morale Scale was completed by the participant with or without help from a family member or friend. Higher scores in the FIM and PGC imply higher QOL and ADL function. When the PGC or FIM could not be administered due to hospitalization or other interruption, or if all items in either evaluation were not completed, we judged that the data were missing and the patient was excluded from analysis.

\subsection{Demographics}

Clinical data and patient background information were collected by creating a personal questionnaire that included age, sex, illness name, course of treatment, level of care required, family structure, employment 
history, education history, hobbies and any participation in religious activities.

\subsection{Statistical Analyses}

The regularity of data distribution was validated using the Shapiro-Wilk test. Comparisons of factors of both the OT and non-OT cohorts were analysed using the Mann-Whitney U test. Correlation analysis between two variables in the PGC and FIM was analysed using the Spearman's $\rho$ (rank correlation coefficient), with the significance of correlation coefficients also examined. Cross-group QOL trends were identified using hierarchical cluster analysis. The significance of probabilities was calculated using Fisher's exact probability test, with significance set at $p<0.05$. Power analyses and statistical calculations were conducted using specialized software (G*Power ver. $3.1{ }^{\circledR}$, Heinrich Heine University; Düsseldorf, Germany). All other analyses were performed using social science software (SPSS ver. 22.0®, IBM; Armonk NY, USA).

\subsection{Consent and Ethical Approval}

All patients who participated in this study gave informed consent in writing. This study received formal ethical approval from Osaka Prefecture University Research Ethics Committee (2012-OT-17).

\section{Results}

\subsection{Reasons for recruitment and exclusion}

Therapy for 100 patients by occupational therapists as well as care for the 100 non-OT patients by nurses and caregivers was initiated out of the 12 home visit stations. Twenty-six patients receiving OT and 38 patients receiving non-OT care could not continue their home healthcare over the study year due to placement in a nursing home, moving to a distant family home, death or other reasons $(53 \%, 17 \%, 14 \%, 9 \%$ and $7 \%$, respectively). The remaining 136 patients (74 in the OT cohort and 62 in the non-OT cohort) were analysed as survey participants.

\subsection{Participant Characteristics}

Mean age (years), years of schooling, FIM score and PGC score of the 200 patients was $78.13 \pm 5.1$, $10.95 \pm 2.3,84.73 \pm 20.4$ and $9.09 \pm 2.6$, respectively, at the time of initiation of the survey. Other information we acquired included degree of nursing care needed, name of injuries and diseases, therapeutic course, occupational history, hobbies, religious observance participation and family configuration (Table 1). On comparison of baseline information between the OT and non-OT cohorts, significant differences between the two cohorts were seen in mean FIM score, with a score of 81 for the OT cohort and 92 for the non-OT cohort $(p=0.024)$ and the presence or absence of a hobby ( $64 \%$ for OT, $47 \%$ for non-OT; $p=0.023)$. No other significant differences between the cohorts were observed.

\subsection{Mean FIM and PGC scores in the OT and non-OT cohorts after 1 year}

Table 2 shows mean changes in FIM and PGC scores at the end of the year of the survey. PGC and FIM scores increased in the OT cohort. The change in mean PGC scores in the non-OT cohort was not significant, while FIM scores decreased significantly over the year.

\subsection{Trajectories of $Q O L$}

Cluster analysis of data over the four visits revealed different trajectories of the subjects' QOL ratings. We labelled these QOL trajectories as follows: A. Improving, B. Worsening, C. Stable Satisfactory, D. Stable Unsatisfactory and E. Slightly Improving. These are illustrated in Fig. 1.

Members of the OT cohort were overrepresented in the favourable trajectories A, C and E $(p<0.00)$ and members of the non-OT cohort were overrepresented in the unfavourable trajectories B and D (Fig. 2).

\subsection{Factors affecting QOL trajectory}

The general characteristics of those following Trajectory A (Improving QOL) were that they started home healthcare immediately after discharge from hospital; they had worked in technical occupations and they tended to have hobbies and participated in religious activities. No specific trend was seen in family structure. A high percentage (93\%) received OT.

The general characteristics of those following Trajectory B (Worsening QOL) were that home healthcare became necessary while they were receiving medical care. Their occupational histories were in sales, business, or transportation and communication. They tended to have no hobbies. No significant differences in religious activities or family configuration were seen. Most Trajectory B patients (63\%) were in the non-OT cohort.

The general characteristics of those following trajectory C (Stable Satisfactory QOL) were that home healthcare became necessary while they were receiving routine outpatient care. Their work histories were in forestry and fishery occupations. They had hobbies and participated in religious activities. Their family configuration was mostly three-generation households, with $40 \%$ in the non-OT cohort.

The general characteristics of those who followed Trajectory D (Stable Unsatisfactory QOL) were that they had occupational histories in manufacturing and 
Table 1. Patient characteristics.

\begin{tabular}{|c|c|c|c|c|}
\hline & & OT cohort & Non-OT cohort & $P$ \\
\hline & Number & 100 & 100 & \\
\hline & Age & $80.0 \pm 7.0$ & $80.0 \pm 9.0$ & 0.969 \\
\hline & Years of education & $12.0 \pm 3.0$ & $12.0 \pm 3.0$ & 0.810 \\
\hline & FIM score & $81.0 \pm 35.0$ & $92.0 \pm 26.8$ & 0.024 \\
\hline & PGCs core & $8.0 \pm 3.0$ & $10.0 \pm 4.0$ & 0.206 \\
\hline \multirow[t]{2}{*}{ Sex } & Women & 60 & 61 & \multirow{2}{*}{1.000} \\
\hline & Men & 40 & 39 & \\
\hline \multirow[t]{5}{*}{ Level of care required } & 1 & 19 & 21 & \multirow[t]{5}{*}{0.857} \\
\hline & 2 & 30 & 30 & \\
\hline & 3 & 20 & 24 & \\
\hline & 4 & 23 & 20 & \\
\hline & 5 & 8 & 5 & \\
\hline \multirow[t]{6}{*}{ Classification of disease } & Musculoskeletal system & 18 & 13 & \multirow[t]{6}{*}{0.870} \\
\hline & Nervous system & 32 & 32 & \\
\hline & Heart or lung disease & 10 & 15 & \\
\hline & Trauma & 17 & 16 & \\
\hline & Systemic disease & 12 & 13 & \\
\hline & Immune disease & 11 & 11 & \\
\hline \multirow[t]{2}{*}{ Course of treatment } & Discharge $\rightarrow$ Home care & 52 & 44 & \multirow[t]{2}{*}{0.322} \\
\hline & Visit $\rightarrow$ Home care & 48 & 56 & \\
\hline \multirow[t]{8}{*}{ Profession } & Office work & 7 & 7 & \multirow[t]{8}{*}{0.588} \\
\hline & Selling & 12 & 7 & \\
\hline & Professional or technical & 18 & 16 & \\
\hline & Service & 1 & 3 & \\
\hline & Agriculture, forestry or fisheries & 26 & 22 & \\
\hline & Production or labour work & 16 & 19 & \\
\hline & Transportation or communication & 2 & 7 & \\
\hline & Others & 18 & 19 & \\
\hline \multirow[t]{2}{*}{ Hobby } & Yes & 64 & 47 & \multirow[t]{2}{*}{0.023} \\
\hline & None & 36 & 53 & \\
\hline \multirow[t]{2}{*}{ Faith } & Yes & 64 & 55 & \multirow[t]{2}{*}{0.249} \\
\hline & None & 36 & 45 & \\
\hline \multirow[t]{4}{*}{ Family structure } & Alone & 26 & 33 & \multirow[t]{4}{*}{0.342} \\
\hline & Couple & 36 & 41 & \\
\hline & Two-family & 19 & 13 & \\
\hline & Three household & 19 & 13 & \\
\hline
\end{tabular}

- $t$-test was used for age, years of education, FIM and PGC

- Fisher's exact test was used for sex, level of care required, classification of disease, course of treatment, profession, hobby, faith, or family structure

Level of care required is according to the Japan's long-term care insurance system, in which $1=$ minimal care requirement category and $5=$ maximal care requirement category. Although the length of convalescence varied, these were categorised into groups by three-month periods, based on the time service provision began.

Table 2. Effect size of OT.

\begin{tabular}{lcccc}
\hline & $\begin{array}{c}\text { Mean 1-year } \\
\text { change in } \\
\text { PGC }\end{array}$ & $p$ & $\begin{array}{c}\text { Mean 1-year } \\
\text { change in } \\
\text { FIM score }\end{array}$ & $p$ \\
\hline OT cohort & 1.92 & 0.001 & 5.07 & 0.001 \\
Non-OT cohort & -0.16 & 0.617 & -6.07 & 0.001 \\
\hline
\end{tabular}

$\mathrm{OT}=$ occupational therapy labour services. They tended not to have hobbies and did not participate in religious activities. Their family configuration was mostly two-generation households, and $43 \%$ were in the non-OT cohort.

The general characteristics of those who followed Trajectory E (Slightly Improving) were that they started home healthcare immediately after discharge from the hospital. Their occupational history was in the service sector. They lived alone and showed no specific trends in hobbies or religious activities. A total of $38 \%$ were in the OT cohort. 


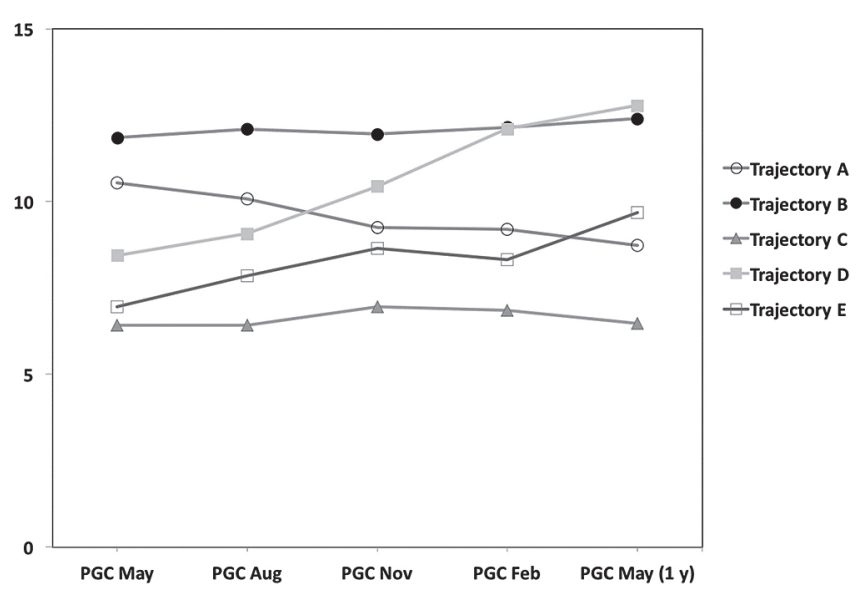

Fig. 1. The five trajectories.

A. Improving QOL (full square), B. Worsening QOL (empty circle), C. Stable Satisfactory QOL (full circle), D. Stable Unsatisfactory QOL (triangle), and E. Slightly Improving QOL (empty square).

PGC score $=$ Philadelphia Geriatric Center Morale Scale score.

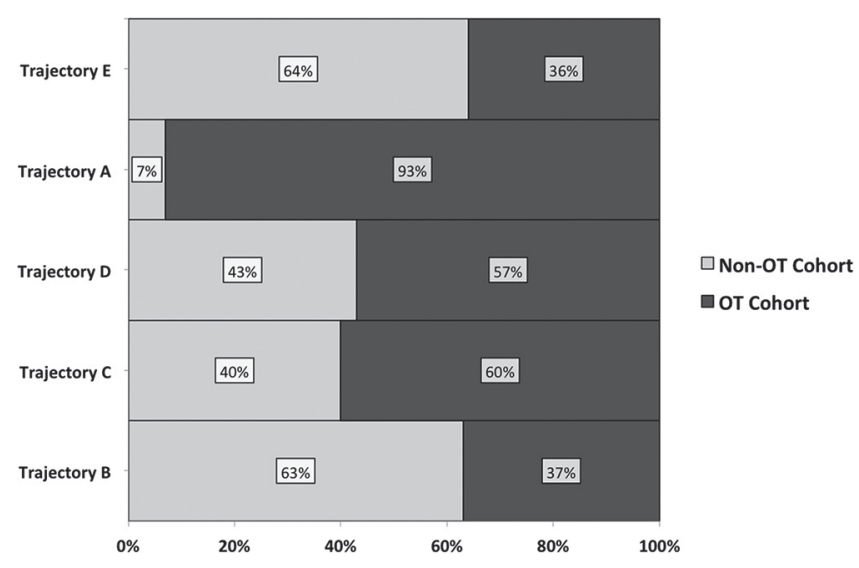

Fig. 2. Proportion of OT and non-OT cohorts in the five trajectories.

Kruskal-Wallis testing identified six factors that correlated with trajectory, namely therapeutic course, specifically the receipt of home care services immediately after discharge, and patient need for both home care service and medical care from their general practitioner; occupational history; hobbies; religious participation; family configuration and OT.

\section{Discussion}

Clients navigating the challenges of ageing face many difficulties, not all of which can be changed, such as family structure. In this study, of the several services offered to patients in their homes, including nursing, help with ADL and OT, we found that OT was the only service that significantly improved QOL. Patients receiving OT who were involved in religious participation and hobbies showed the most improvement. The importance of hobbies has been noted by others [12]; this is an easy feature to implement and may be combined with OT. Religious participation cannot be mandated, but an increased presence of clergy where the elderly congregate should certainly be encouraged. All three of these factors could be implemented wherever there is group participation, such as in community centres. Nevertheless, it is important to emphasize that the greatest need is found among those who live alone and are isolated. Another problem that needs to be addressed is the issue of help with ADL. Mandated bathing and meal times, arranged according to the health centre personnel's schedule, may promote passivity and lower the patient's feelings of independence. This will have to be addressed in creative ways. By becoming familiar with the different trajectories of the different groups, OT and also ADL personnel may be able to tailor their services to the patients' needs, working together to improve independence and QOL.

Several limitations of the study warrant mention. First, our study was confined to a part of the Kansai district, and the experiences and QOL of Japanese in other areas may differ. Second, the nature of our study's inclusion criteria almost guaranteed small subject numbers regardless of location. Finally, we did not investigate how OT should be tailored to meet the needs of patients who face different trajectories.

\section{Conclusions}

The fact that receivers of OT were under-represented in the lower QOL cohorts is encouraging; it implies that the kind of OT provided currently has a positive effect on elderly individuals. Further work on the implementation of hobby activity, possibly in the context of OT, may be fruitful. This was a small study, but with interesting findings. We hope it will encourage larger studies of OT in the rapidly expanding elderly population of Japan.

\section{References}

[1] Cabinet Office, Government of Japan: Heisei 24 aged society white paper [last accessed 2015 June 1] Available from http:/www8.cao.go.jp/kourei/whitepaper/w-2014/ zenbun/pdf/1s1s_5.pdf, 2015.6.1.

[2] Campbell JC, Ikegami N. Long-term health insurance comes to Japan. Health Affairs. 2000; 19: 26-39. Doi: 10.1377/hlthaff.19.3.26.

[3] Oe M. Problems and implications of Japan's ageing 
society for future urban developments. Policy and Governance Working Paper Series. The 21st Century Center of Excellence Program. Graduate School of Medicine and Governance, Keio University, Japan. Delivered March 2006.

[4] Yamada S, Hanagama M, Kobayashi S, Satou H, Tokuda S, Niu K, et al. The impact of the 2011 Great East Japan Earthquake on hospitalization for respiratory disease in a rapidly ageing society: A retrospective descriptive and cross-sectional study at the disaster base hospital in Ishinomaki. BMJ Open. 2013; 3: e000865. Doi: 10.1136/ bmjopen-2012-000865.

[5] Muramatsu N, Akiyama H. Japan: Super-ageing society preparing for the future. The Gerontologist. 2011; 51: 425-32. Doi: 10.1093/geront/GNR067.

[6] Tsutsui T, Muramatsu N. Japan's universal long term care system reform of 2005: Containing costs and realizing a vision. Journal of the American Geriatrics Society. 2007; 55: 1458-63. Doi: 10.1111/j.1532-5415.2007.01281.x.

[7] Kawai H. "Oiru” tohadouiukotoka, 24th edition. Tokyo: Kodansha, (in Japanese). 2011.

[8] Washida K. Oinokuuhaku, 3rd edition, Tokyo: Koubundou, [in Japanese]. 2003.

[9] Lawton MP. The Philadelphia Geriatric Center Morale Scale: A revision. Journal of Gerontology. 1975; 30: 85-9.

[10] Liang J, Bollen KA. Sex differences in the structure of the Philadelphia Geriatric Center Morale Scale. Journal of Gerontology. 1985; 42: 468-77.

[11] Koyano W. Studies in Japanese social gerontology. Journal of the National Institute of Public Health. 2004; 53: 204-8.

[12] Iwasa H, Yoshida Y, Suzuki T, Kim H, Yoshida H. Leisure activities and cognitive function in elderly community-dwelling individuals in Japan: A 5-year prospective cohort study. Journal of Psychosomatic Research. 2012; 72: 159-64. Doi: 10.1016/j.jpsychores.2011.10.002.

\section{APPENDIX}

Contents of the PGC-MS instrument

Answers are given as yes or no and scored as one point for yes and zero points for no, for a maximum of 17 points.

1. Things keep getting worse as I get older.

2. I have as much pep as I had last year.

3. I feel lonely.

4. Little things bother me more this year.

5. I see enough of my friends and relatives.

6. As you get older, you are less useful.

7. I sometimes worry so much that I can't sleep.

8. As I get older, things are (better/worse) than I thought they would be.

9. I sometimes feel that life isn't worth living.

10. I am as happy now as I was when I was younger.

11. I have a lot to be sad about.

12. I am afraid of a lot of things.

13. I get mad more than I used to.

14. Life is hard for me much of the time.

15. I am not satisfied with my daily life.

16. I take things hard.

17. I get upset easily. 